\title{
Endocrine-disrupting chemicals and antibiotics in the Pearl River Delta and coastal environment: sources, transfer, and implications
}

\author{
WEIHAI XU ${ }^{1}$, WEN YAN ${ }^{1}$
}

${ }^{1}$ Key Laboratory of Ocean and Marginal Sea Geology, South China Sea Institute of Oceanology, Chinese Academy of Sciences, Guangzhou 510301, China

Endocrine-disrupting chemicals (EDCs) and antibiotics were investigated in riverine runoff of the Pearl River Delta (PRD) and Pearl River Estuary (PRE), South China for assessing the importance of riverine runoff in the transportation of contaminants from terrestrial sources to the open ocean. The EDCs detected in eight outlets of the Pear River and the Pear River Estuary (PRE) were in the ranges of 1.2-234 ng/L and $0.2-178 \mathrm{ng} / \mathrm{L}$, respectively. The estrogen equivalents in the aquatic environments under study ranged from 0.08 to $4.5 \mathrm{ng} / \mathrm{L}$, with $\mathrm{E} 1$ and EE2 being the two predominant contributors. All antibiotics were detected in the eight outlets with concentrations ranging from 0.7 to 127 $\mathrm{ng} / \mathrm{L}$. As the fluxes of the EDCs from the PRD region to the nearby ocean are over 500 tons each year. The figure for the antibiotics was 193 tons with 102 tons from the fluoroquinolone group. It showed that antibiotics decreased from the riverine outlets to the PRE and open ocean. Risk assessment showed that most of these antibiotics showed various ecological risks to the relevant aquatic organisms, in which ofloxacin (OFL), erythromycin (ETM) and ciprofloxacin (CIP) posed high ecological risks to the studied aquatic environments. 